\title{
Evidence of SARS-CoV2 Entry Protein ACE2 in the Human Nose and Olfactory Bulb
}

\author{
Moritz Klingenstein $^{a} \quad$ Stefanie Klingenstein ${ }^{a}$ Peter $\mathrm{H}$. Neckel ${ }^{b}$ \\ Andreas F. Mack ${ }^{\mathrm{b}}$ Andreas P. Wagner ${ }^{\mathrm{b}}$ Alexander Kleger ${ }^{\mathrm{c}}$ Stefan Liebau $^{\mathrm{a}}$ \\ Alfio Milazzo ${ }^{\text {a }}$ \\ anstitute of Neuroanatomy and Developmental Biology, Eberhard Karls University Tübingen, Tübingen, Germany; \\ bInstitute of Clinical Anatomy and Cell Analysis, Eberhard Karls University Tübingen, Tübingen, Germany; \\ 'Department of Internal Medicine I, University Medical Center UIm, Ulm, Germany
}

\section{Keywords}

SARS-CoV2 - ACE2 - Human - Olfactory epithelium ·

Olfactory bulb

\begin{abstract}
Usually, pandemic COVID-19 disease, caused by SARS-CoV2, presents with mild respiratory symptoms such as fever, cough, but frequently also with anosmia and neurological symptoms. Virus-cell fusion is mediated by angiotensin-converting enzyme 2 (ACE2) and transmembrane serine protease 2 (TMPRSS2) with their organ expression pattern determining viral tropism. Clinical presentation suggests rapid viral dissemination to the central nervous system leading frequently to severe symptoms including viral meningitis. Here, we provide a comprehensive expression landscape of ACE2 and TMPRSS2 proteins across human postmortem nasal and olfactory tissue. Sagittal sections through the human nose complemented with immunolabelling of respective cell types represent different anatomically defined regions including olfactory epithelium, respiratory epithelium of the nasal conchae and the paranasal sinuses along with the
\end{abstract}

karger@karger.com

(c) 2021 S. Karger AG, Basel

www.karger.com/cto

Karger! hardly accessible human olfactory bulb. ACE2 can be detected in the olfactory epithelium as well as in the respiratory epithelium of the nasal septum, the nasal conchae, and the paranasal sinuses. ACE2 is located in the sustentacular cells and in the glandular cells in the olfactory epithelium as well as in the basal cells, glandular cells, and epithelial cells of the respiratory epithelium. Intriguingly, ACE2 is not expressed in mature or immature olfactory receptor neurons and basal cells in the olfactory epithelium. Similarly, ACE2 is not localized in the olfactory receptor neurons albeit the olfactory bulb is positive. Vice versa, TMPRSS2 can also be detected in the sustentacular cells and the glandular cells of the olfactory epithelium. Our findings provide the basic anatomical evidence for the expression of ACE2 and TMPRSS 2 in the human nose, olfactory epithelium, and olfactory bulb. Thus, they are substantial for future studies that aim to elucidate the symptom of SARS-CoV2 induced anosmia via the olfactory pathway.

c) 2021 S. Karger AG, Basel
Stefanie Klingenstein

Institute of Neuroanatomy and Developmental Biology Eberhard Karls University Tübingen Oesterbergstr. 3, DE-72074 Tübingen (Germany) stefanie.klingenstein@uni-tuebingen.de 


\section{Introduction}

The Coronavirus Disease 2019 (COVID-19) emerged from East Asia and quickly spread all over the world reaching a pandemic scale [Guan et al., 2020]. The infiltration of the virus SARS-CoV2 into different cell types leads to different symptoms with varying severity. According to clinical studies worldwide, the most prevalent symptoms are fever, cough, fatigue, headache, dyspnea, sputum production, arthralgia, diarrhea, rhinorrhea, and sore throat [Krajewska et al., 2020; Zhou et al., 2020]. Since many patients also report olfactory and gustatory

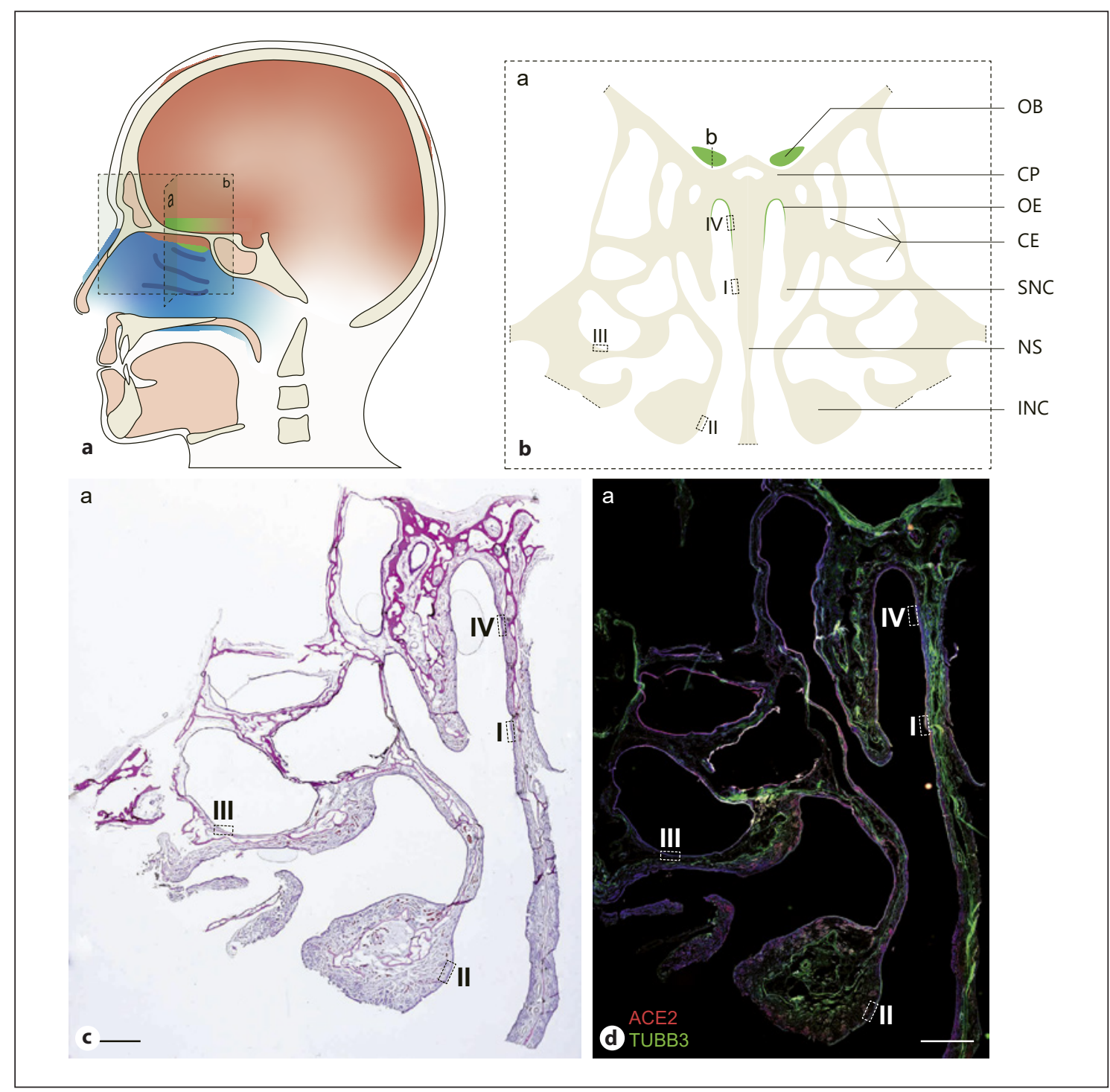

Fig. 1. a Schematic illustration of a human head. Frontal section highlights the nose specimen (a). Sagittal view of the olfactory bulb section (b). Olfactory epithelium and olfactory bulb, both marked in green, are located at the upper nasal cavity and above the cribriform plate. b Schematic representation of the nose specimen from the frontal section through the head (a). The olfactory epithelium (OE) and the olfactory bulb (OB) are shown in green. Section plane for better comprehensibility of the olfactory bulb (b). The dashed boxes show the section of the respiratory epithelium of the nasal septum (NS) (l), the intermediate nasal conchae (INC)
(II), the cellulae ethmoidales (CE) (III) and the olfactory epithelium (OE) (IV). CP, cribriform plate; SNC, superior nasal conchae. c. Hematoxylin eosin staining of the right half of the nose specimen. Dashed boxes show the same areas found in the schematic picture in $\mathbf{b}$. d Immunofluorescence staining of the right half of human nose specimen. TUBB3 is shown in green, ACE2 in red, nuclei in blue. Dashed boxes show the same areas found in the schematic picture in $\mathbf{b}$. More details for area I, II, III of the respiratory epithelium can be found in Fig. 2. More details for area IV olfactory epithelium can be found in Fig. 3. Scale bar, $2.5 \mathrm{~mm}$. 


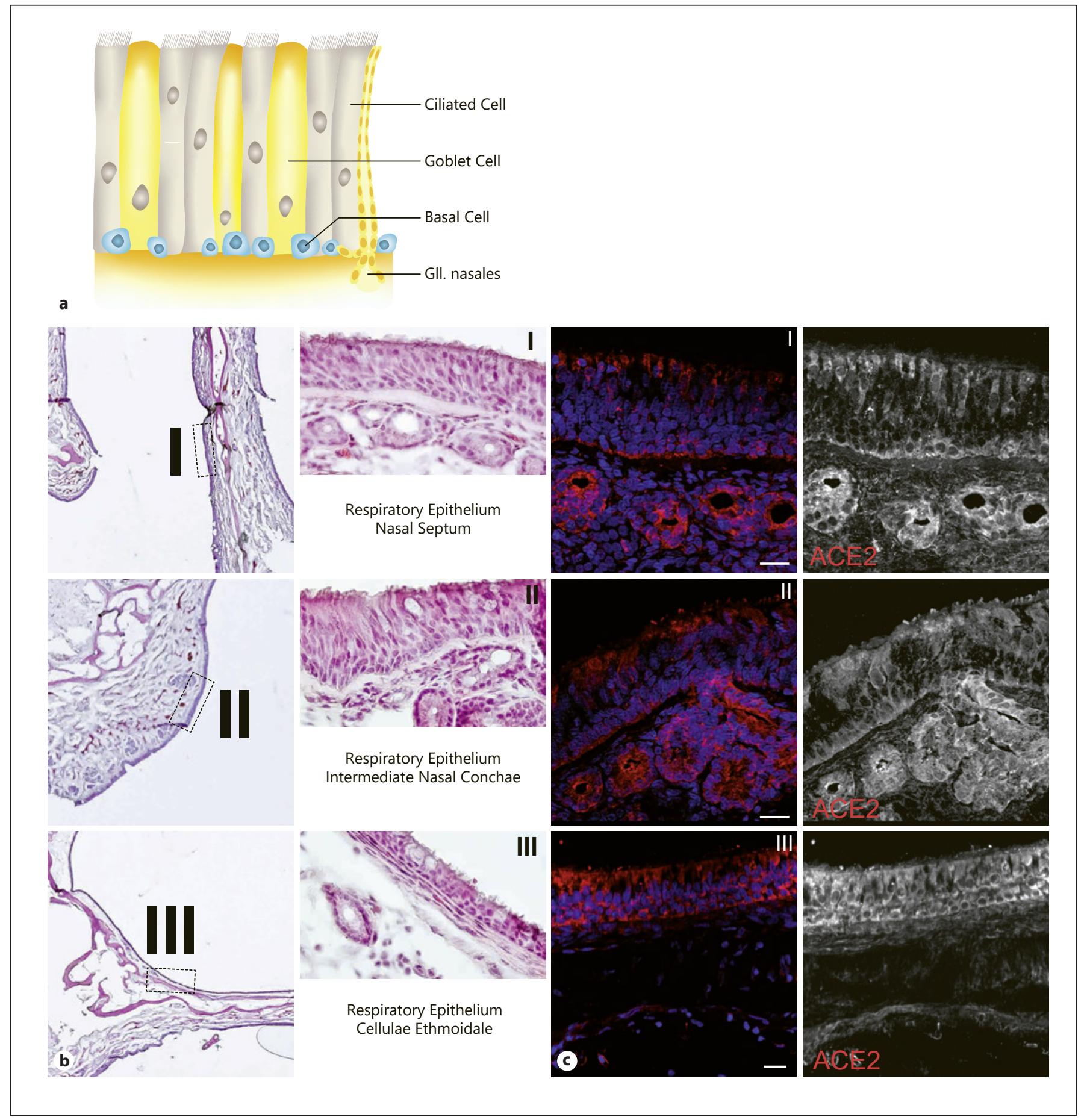

Fig. 2. a Schematic illustration of the pseudostratified respiratory epithelium with ciliated cells, goblet cells, basal cells, and nasal submucosal glands. b Hematoxylin eosin stainings of the respiratory epithelium in the area of the nasal septum (l), the intermediate nasal conchae (II), and the cellulae ethmoidales (III). c Immunofluorescent stainings of the respiratory epithelium verified by absent TUBB3 expression in the epithelium from dashed boxes I, II and III. TUBB3 is a highly specific marker for mature and immature ORN. ACE2 (red) positive basal cells of the respiratory epi- thelium of the nasal septum as well as positive apical staining of respiratory epithelial cells and nasal submucosal glands (1). ACE2 (red) protein expression is located in the ciliated epithelium cells and the basal cells of the respiratory epithelium of intermediate nasal conchae as well as in the underlying nasal submucosal glands (II). ACE2 (red) expression in the cellulae ethmoidales can be found in epithelial cells and basal cells (III). Nuclei are shown in blue. Scale bar, $20 \mu \mathrm{m}$. 
dysfunctions, these symptoms are considered typical of the SARS-CoV2 infection [Lechien et al., 2020; Lee et al., 2020]. There are many steps involved in the perception of smell where the infection with SARS-CoV2 could potentially be the cause of anosmia, starting with the transport of the odorants to the receptors in the olfactory neurons extending to the signal transduction to different olfactory cortex areas.

In the olfactory epithelium, a variety of histological target structures, including the olfactory receptor neurons (ORN) with their ensheathed axons, sustentacular, microvillar or glandular cells could serve as a viral target and therefore influence olfactory function. Another area of viral attack could be the olfactory bulb. Here, the fila olfactoria or projection neurons in different layers of the olfactory bulb could be targeted by the virus, causing disruption in olfactory perception.

Infection of host cells with SARS-CoV-2 is preceded by a complex process of virus attaching, receptor recognition and proteolytic cleavage of the transmembrane spike glycoprotein to promote virus-cell fusion mediated by angiotensin-converting enzyme 2 (ACE2) and transmembrane serine protease 2 (TMPRSS2) [Hoffmann et al., 2020].

Up to date, there is no clear evidence which cell types of the olfactory and respiratory epithelium express ACE2 and TMPRSS2. Transcriptional but also data from murine and human olfactory tissue show as of now report conflicting data [Bilinska et al., 2020; Brann et al., 2020; Hou et al., 2020; Ueha et al., 2020]. In addition, the reports on localization of the viral entry proteins in different cell types from the epithelia vary [Bilinska et al., 2020; Ueha et al., 2020]. Protein expression was found in sustentacular cells in all publications, but only one paper claims a low ACE2 expression in ORN [Ueha et al., 2020]. TMPRSS2 was found in murine and human respiratory

Fig. 3. a Schematic structure of human olfactory epithelium. The mature olfactory receptor neurons are highlighted in green and are surrounded by sustentacular cells. The sensory neurons arise via an olfactory precursor step from basal cells. There are 2 types of basal cells: horizontal and globose basal cells. Bowman glands are secretory glands that are found exclusively in the olfactory epithelium. b Hematoxylin eosin staining of the olfactory epithelium in the area of the nasal septum (IV). c Immunofluorescent staining of the olfactory epithelium from dashed box (IV). Merged picture shows ACE2 in red, OMP, a marker for mature ORN, in white and TUBB3, a marker for mature and immature ORN, in green. Positive ACE2 staining is exclusively located to the region of the sustentacular cells, no appearance in basal cells or co-localization with OMP or TUBB3. d Enlarged picture from the olfactory epithelium epithelium and murine olfactory epithelium [Bilinska et al., 2020]. Similarly, experiments performed in the murine olfactory bulb showed partly contradictory results for ACE2 staining. Until now, there are no protein verifications in human olfactory bulb due to its difficult accessibility.

Here, we employ a unique human postmortem tissue resource to thoroughly study the ACE2 and TMPRSS2 protein expression patterns in the human olfactory system, including olfactory epithelium, respiratory epithelium of the nasal septum, the nasal conchae, and the paranasal sinuses as well as the olfactory bulb. These findings will help to explain the symptom of anosmia as well as frequent dissemination to the central nervous system in COVID-19 patients and give a starting point to further investigations on how SARS-CoV2 can affect the olfactory system.

\section{Materials and Methods}

\section{Tissue Processing}

The tissue was obtained from human body donors. Surgery was performed transcranial with particular attention to remove the cribriform plate together with the olfactory bulb as well as the nasal septum and the nasal conchae. Fixation of the whole specimen was performed for 4 days with daily changes of Roti Histofix (Carl Roth, P087.1) fixation media, followed by 1-day washing step with PBS. Decalcification was achieved with 10\% EDTA for 70 days with medium change twice a week. After a washing step for 1 day with PBS, the specimen was incubated for another day in $30 \%$ sucrose, followed by embedding with Tissue-Tek (Thermo Fisher, 12351753) for frozen sections.

\section{Immunohistological Staining}

For hematoxylin-eosin staining, $12 \mu \mathrm{m}$ frozen sections were treated with filtered hematoxylin (Sigma, H9627) for $10 \mathrm{~min}$, followed by a washing step with tap water for 2-5 min. Eosin (Sigma, 230251) counterstaining was performed for $7 \mathrm{~min}$, followed by careful rinses with distilled $\mathrm{H}_{2} \mathrm{O}$ and dehydration with increasing

and mucosa showing ACE2 positive sustentacular cells and positive Bowman glands. Upper dashed box shows an enlarged picture of ACE2 positive sustentacular cell, marked with dashed white lines, but negative for TUBB3. TUBB3 positive olfactory sensory neuron marked with dashed yellow lines. Lower dashed box shows ACE2 positive staining in Bowman glands in the olfactory submucosa. One ACE2 positive Bowman gland cell is marked with a dashed white line. e Immunofluorescent picture with TMPRSS2 (green) and OMP (red) expression in the olfactory epithelium (IV). TMPRSS2 is mainly located in the sustentacular cells and shows a minor expression in Bowman glands, but no expression in basal cell or OMP-positive mature ORN. Nuclei are shown in blue. Scale bar, $20 \mu \mathrm{m}$.

(For figure see next page.) 


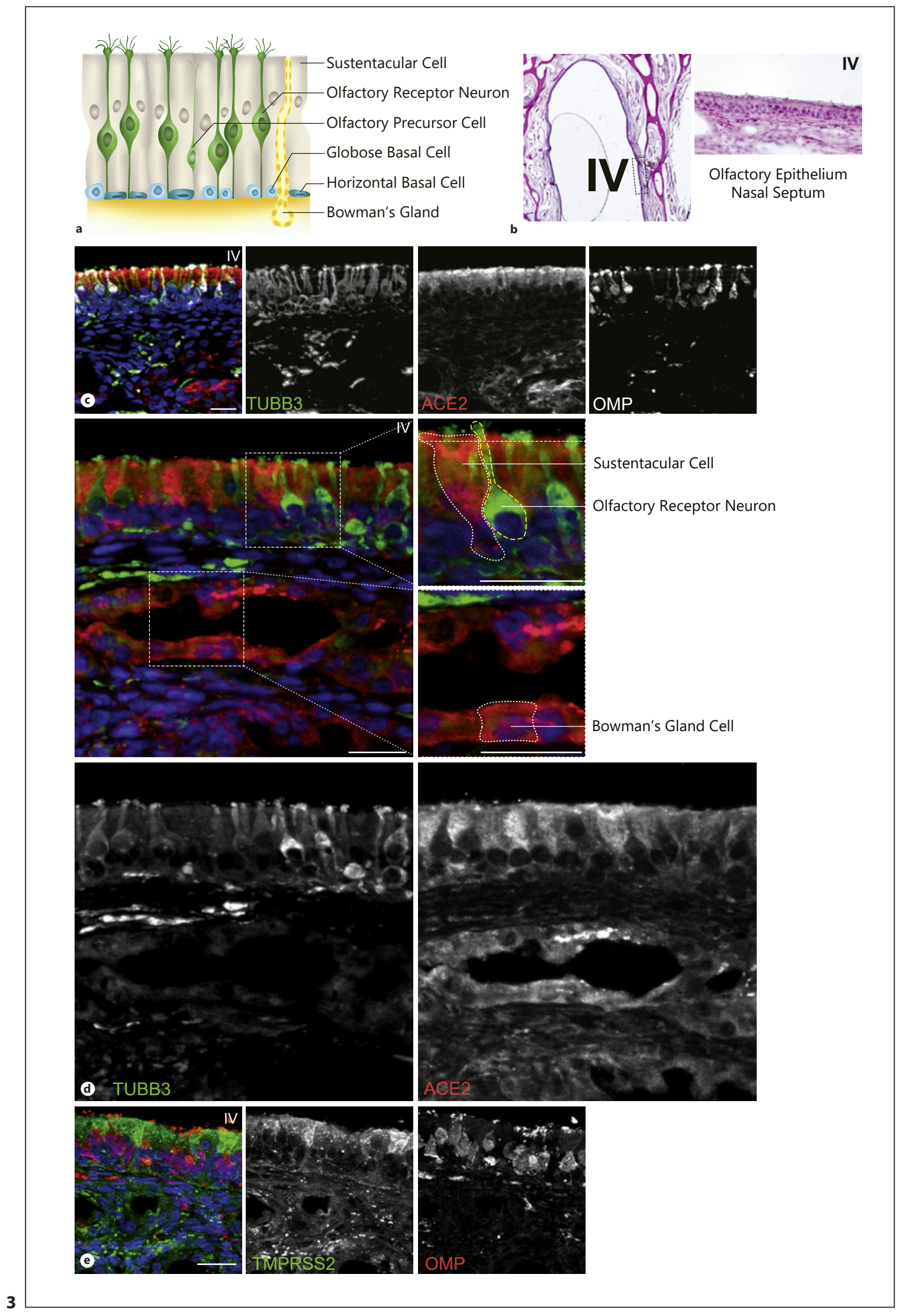


alcohol concentrations (70,95, and 100\%). After washing 2 times in xylene, sections were mounted with DPX mounting media (VWR, 13,514).

\section{Immunofluorescence Staining}

For immunofluorescence staining, $12 \mu \mathrm{m}$ frozen sections were rehydrated for 5 min with PBS (Thermo Fisher, 10010056) followed by an ethanol gradient each $30 \mathrm{~s}(70,95,99,95$, and $70 \%)$. The frozen sections were blocked in skimmed milk blocking buffer (TBS $+10 \%$ NDS $+1.25 \%$ BSA, $4 \%$ skimmed milk, $0.1 \%$ Triton $\mathrm{X}$ ) for $30 \mathrm{~min}$ at room temperature. The primary antibody was diluted in skimmed milk blocking solution and incubated over night at $4^{\circ} \mathrm{C}$. The following primary antibodies were used: ACE2 $\mathrm{rb}(\mathrm{Ab}-$ cam, ab15348 1:100), glial fibrillary acidic protein (GFAP) ms (Merck, MAB360, 1:100), TMPRSS2 ms (Santa Cruz, Sc515727, 1:50), BIII-tubulin (TUBB3) ms (BioLegend, 802002, 1:1000), and olfactory marker protein (OMP) gt (WAKO, 544-1001 1:500). Synaptophysin rb (Abcam, 14,692-100, 1:100). All primary antibodies were verified in appropriate tissue (online suppl. Fig 1; see www.karger.com/doi/10.1159/513040). After several washing steps, secondary antibodies were diluted 1:100 in PBS together with DAPI (Abcam, ab228549) and incubated for $45 \mathrm{~min}$ in room temperature away from daylight. The following secondary antibodies were used: Darb 488 (Invitrogen, A32790), Dams 488 (Invitrogen, A32766), Darb 546 (Invitrogen, A10040), Dagt 546 (Invitrogen, A11056), and Dams 647 (Abcam, ab150107). Sections were embedded with Mowiol (Carl Roth, 0713). Immunofluorescence stainings were analyzed using the Axio Imager. M2 microscope with the AxioVision software (Zeiss).

Scans of immunolabelled nasal specimen were done by order of Zeiss, pictures were analyzed with ZEN Blue software (Zeiss).

\section{Results}

Anatomical Regions Analyzed in Human Postmortem Nasal and Olfactory Tissue

High ACE2 and TMPRSS2 expressions have been documented in the respiratory, gastrointestinal, and reproductive system [Fan et al., 2020; Ren et al., 2020]. Focusing on the upper respiratory tract, increased ACE2 and

Fig. 4. a Schematic illustration of the human olfactory bulb with its layers: Under most is the outer nerve layer with the axons of the olfactory receptor neurons followed by the glomerular layer with olfactory glomerula connecting the axons with interneurons. Synaptic processing between the glomerular layer and the mitral cell layer occurs in the external plexiform cell layer. In the mitral cell layer, the mitral cells are located. They form synapses in the inner plexiform layer with the granular cells of the granular cell layer. b Hematoxylin eosin staining of a sagittal section of the human olfactory bulb (b). Dashed box shows an enlarged picture in (c). Scale bar, $2.5 \mathrm{~mm}$. c Immunofluorescence staining of human olfactory bulb. Synaptophysin is shown in green. The specific layers of the olfactory bulb are marked. ONL, outer nerve layer; GL, glo-
TMPRSS2 are found in the respiratory and olfactory mucosa. First, a frontal section of human postmortem tissue through the nose with distinct anatomical regions (shown in Fig. 1a) is examined. The olfactory epithelium (IV) is located at the roof of the nasal cavity between the nasal septum and the superior nasal conchae. The paranasal sinuses, in particular the cellulae ethmoidales (III), are located next to the nasal turbinate. In addition, the nasal septum (I), the superior nasal conchae, and the intermediate nasal conchae (II), all of which covered with respiratory epithelium, are present in this section (shown in Fig. 1b). With hematoxylin-eosin staining, these different regions of the nasal cavity were identified and visualized (shown in Fig. 1c, 2b, 3b). Overview of ACE2 and TUBB3 immunolabelling in postmortem nasal tissue (shown in Fig. 1d). TUBB3, a marker for mature and immature olfactory sensory neurons allows distinguishing the olfactory epithelium from the other non-neural areas within the nose specimen (Fig. 1d).

\section{Identification of ACE2 in Different Areas of the Respiratory Epithelium}

Schematic illustration and hematoxylin-eosin staining show the anatomical stratification of the analyzed regions of the nasal cavity (Fig. 1c, 2a, b). The different areas of the respiratory epithelium, namely the nasal septum (I), the intermediate nasal conchae (II), and the cellulae ethmoidales (III) show pseudostratified ciliated epithelial cells arising from a basal cell layer. Mucus-producing goblet cells can be found in the respiratory epithelium of all 3 areas (Fig. 2b). In the respiratory epithelium of the nasal septum, the intermediate nasal conchae and the paranasal sinus, ACE2 expression was located in epithelial cells as well as in basal cells. High expression was found in the nasal submucosal glands underneath the respiratory epithelium. The ACE2 expression can be found uni-

merular layer; EPL, external plexiform layer; MCL, mitral cell layer; IPL, internal plexiform layer; GCL, glomerular cell layer. d Overview picture with immunofluorescence stainings for ACE2 (green), TUBB3 (red), and OMP (white) illustrating the different layers of the olfactory bulb. The white dashed box indicates the magnified area illustrated in the next image, showing representative ACE2 positive cells, not co-localizing with TUBB3 or OMP. High protein expression of TUBB3 and OMP can be found in the outer nerve layer, whereas ACE2 is mainly located in the glomerular layer. e Picture of the olfactory bulb showing ACE2 (green) and glial marker GFAP (red) positive cells. Some GFAP positive glia cells co-localize with ACE2. Nuclei are shown in blue. Scale bar, 20 $\mu \mathrm{m}$.
(For figure see next page.) 
formly in the gland cells in the submucosa as well as in the excretory part of the nasal glands, localized within the epithelium (Fig. 2c).

Identification of ACE2 and TMPRSS2 in the Olfactory Epithelium

Schematic illustration of the olfactory epithelium (Fig. 3a) and histological staining of the same area (Fig. 3b) show primary sensory neurons that are embedded between the supporting cells. These sustentacular cells provide mechanical strength to the epithelium, generate the olfactory binding protein, and support the other cells with nutrients [Choi and Goldstein, 2018]. Additionally, sustentacular cells are responsible for the maintenance of the ion and water balance within the olfactory epithelium [Suzuki et al., 2000]. ORN arise from basal stem cells via

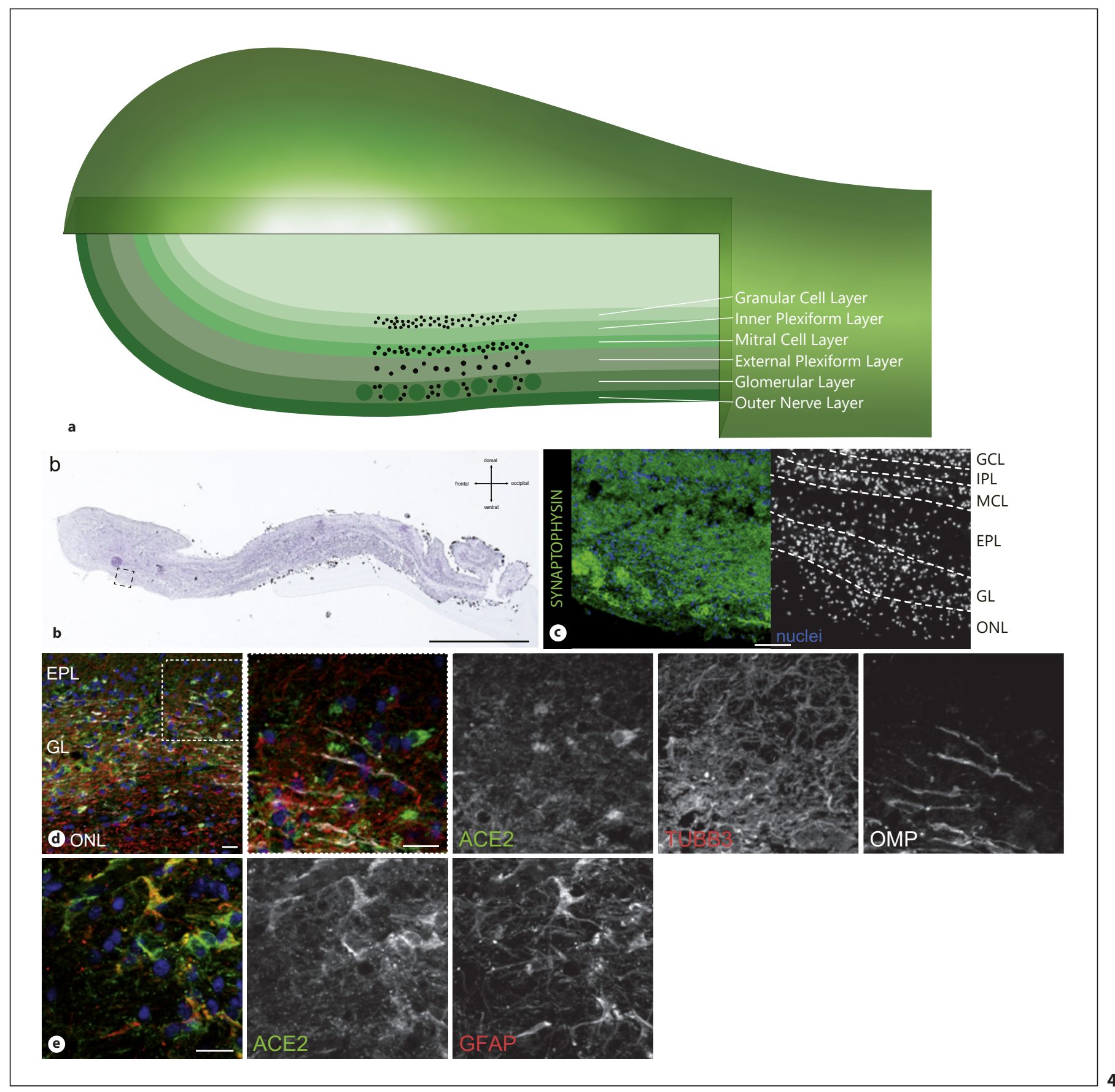

Detection of ACE2 in Human Olfactory

Cells Tissues Organs 2020;209:155-164 DOI: $10.1159 / 000513040$ 
immature and intermediate steps [Caggiano et al., 1994; Fletcher et al., 2017]. Moreover, microvillar cells and excretory ducts from the specialized Bowman glands beneath the epithelium lie scattered in the epithelium [Getchell and Getchell, 1992; Miller et al., 1995]. In addition, the data confirmed the expression of ACE2 in the olfactory epithelium (Fig. 3c, d). The ACE2 staining was mainly located in the supporting cells and could not be found in basal cells, immature ORN, marked with BIIItubulin (TUBB3) nor in mature ORN, marked with olfactory marker protein (OMP) (Fig. 3c). In the underlying lamina propria, high concentrations of ACE2 were found in the Bowman gland cells. ACE2 expression is mainly limited to the sustentacular cells and shows no co-localization with adjacent TUBB3 positive sensory neurons (Fig. 3d). TMPRSS2 was also expressed in the supporting cells of the olfactory epithelium and in the glandular cells of both epithelia, but no expression in the other cell types (Fig. 3e).

\section{Identification of ACE2 in the Olfactory Bulb}

To complete our view of ACE2 expression in the olfactory system, stainings of the human olfactory bulb were performed. Axons from the ORN pervade through the lamina cribrosa to the olfactory bulb. Here, the first synaptic interconnections to the downstream secondary neurons appear [Nagayama et al., 2014]. These defined interconnections are located in 6 distinct layers of the olfactory bulb (Fig. 4a, c). With hematoxylin-eosin staining, these different regions of the olfactory bulb were identified and visualized (Fig. $4 \mathrm{~b}$ ). The processed information projects along the olfactory tract to different regions of the olfactory cortex [Scott et al., 1993]. The hallmark feature of the outer nerve layer is the ensheathed fila olfactoria coming from the olfactory epithelium, penetrate the bone plate and enter the olfactory bulb. Different cell types and their interconnections, like interneurons and projection neurons are located in deeper layers of the olfactory bulb and lead to processed and fine-tuned sensory information (Fig. 4a) [Au et al., 2002]. The olfactory bulb has been analyzed on RNA and on protein level with mouse tissue so far [Brann et al., 2020; Ueha et al., 2020]. In the human olfactory bulb, ACE2 could be found widely distributed, with high expression in the glomerular layer (Fig. 4d). Faint stainings of ACE2 in the mitral cell layer were also detected. However, no co-localization of ACE2 and OMP or TUBB3 could be detected, suggesting that ACE2 is not expressed in the axons of the ORN or other neurons (Fig. 4d). While neurons in the olfactory bulb lack the expression of ACE2, the protein could be localized in some glial fibrillary acidic protein (GFAP)positive glia cells (Fig. 4e), which is consistent with the findings in other brain regions [Deffner et al., 2020].

\section{Discussion and Conclusion}

In our present work, we show that both virus entry proteins, ACE2 and TMPRSS2, are found in the sustentacular cells and Bowman glands of the olfactory epithelium (IV), but not in the primary sensory neurons. In the respiratory epithelium from the nasal septum (I), the intermediate nasal conchae (II), and the cellulae ethmoidales (III), ACE2 expression was detected in the basal cell layer and in the apical part of the respiratory epithelial cells as well as in the nasal submucosal glands. We conclude, based on our findings, that SARS-CoV2 may bind to ACE2 and TMPRSS2 in epithelial cells of the respiratory, olfactory, and paranasal sinus epithelium and can thus penetrate the upper respiratory system. In the olfactory bulb, ACE2 was widely distributed with the highest expression in the glomerular layer, but was not co-localized with OMP positive neurons or other neuronal cell types.

Taken all findings together, this leads to the question, how the symptoms of anosmia can be explained in COVID-19 patients. It could be hypothesized that anosmia in COVID-19 is caused by viral infection of ORN, which further leads to their damage. Corrupted ORN cannot process and project odorant information to the brain via the olfactory bulb and odorant information cannot be processed and projected to the brain via the olfactory bulb. However, in our work we can clearly demonstrate that there is no expression of ACE2 in the primary neurons, which supports results from previous work [Brann et al., 2020; Chen et al., 2020]. Probably, the sustentacular cells are affected by the SARS-CoV2 virus, as these cells express both proteins ACE2 and TMPRSS2. Sustentacular cells are essential for the olfactory system. They do not only provide structural stability comparable to glia cells, but also support all other cells of the epithelium in a nutritious and metabolic way as they are connected via tight and adherens junctions with the other cell types [Suzuki et al., 2000; Steinke et al., 2008; Bilinska et al., 2020]. Moreover, sustentacular cells perform phagocytosis and are probably involved in protective mechanisms by expressing antiviral and antibacterial proteins [Suzuki et al., 1996]. Sustentacular cells are in close contact to the ORN, forming intercellular connections. Potentially, infected sustentacular cells may also invade ORN across these bridges, unassisted by the virus entry genes. 
How does the localization of ACE2 and TMPRSS2 in Bowman gland cells and nasal submucosal glands of the respiratory epithelium fit to our hypothesis? Glandular cells in the nasal cavity, together with goblet cells, which are found in respiratory epithelium, are necessary for the production of mucus. Mucus is a viscous solution, which contains mainly water, ions, proteins and mucins and covers the apical side of the nasal epithelium [Escada et al., 2009]. It helps to maintain the physiological barrier of the epithelium against foreign substances from the air. In particular, the Bowman glands are supposed to produce enzymes providing xenobiotic-metabolizing functions [Renne et al., 2007]. Due to the virus entry protein expression in the Bowman gland and nasal submucosal glands, SARS-CoV2 could destroy many glandular cells which would probably result in a reduced or incorrect mucus production, leading to dysfunction or even damage of ORN. In addition, sustentacular cells and Bowman glands are supposed to produce odorant-binding proteins (OBPs), which are indispensable for the perception of odorants [Vogt et al., 2002; Nagnan-Le Meillour et al., 2019]. Without OBPs in the mucus of the olfactory epithelium, the binding of odorants to the olfactory receptors is hampered.

That in mind, we hypothesize that a disruption of high numbers of sustentacular cells as well as mucus producing glandular cells could lead to a decreased perception of smell and leave the olfactory epithelium less protected against other viral or bacterial threats. The stem cells of the olfactory epithelium, the basal cells, are most probably not affected by COVID-19 infections, maintaining the potential of reproducing ORN, sustentacular cells as well as Bowman glands [Maddux et al., 1993]. This could explain the relatively fast recovery in most patients suffering from COVID-19 triggered anosmia [Hopkins et al., 2020; Lee et al., 2020]. However, some cases have been reported with very slow or nearly no recovery of anosmia [Kosugi et al., 2020]. Depending on the dimension of destruction of sustentacular and glandular cells, reproduction from basal cells followed by recovery of the nasal mucus may be prolonged or, as in severe cases, the destruction of sustentacular cells may also affect the basal cells leading to extended symptoms. In this context, it is noteworthy that we also found ACE2 expression in the basal cells of the respiratory epithelium.

Based on our findings, we presume that SARS-CoV2 can enter the cells from the upper respiratory system via the viral entry proteins ACE2 and TMPRSS2. The infection of the sustentacular cells of the olfactory epithelium together with the underling Bowman gland cells may lead to altered mucus production, metabolism and structural instability in the olfactory epithelium. In addition, infection may result in the inability of the ORNs to connect to odorants via OBPs. Nevertheless, most patients regain their ability of smell perception, due to the fact that the basal cells are presumably not affected by the virus and can therefore replace destroyed cells of the olfactory epithelium.

\section{Statement of Ethics}

Sampling of human material from body donors and all following experiments were made in accordance to local laws and regulations approved by the responsible ethical committee at the Medical Department of the University of Tübingen (Project Nr. 284/2020BO2). The body donors gave their informed consent in concert with the declaration of Helsinki to use the cadaver for research purposes. The procedure was approved by the ethics commission at the Medical Department of the University of Tübingen (Project Nr. 237/2007 BO1).

\section{Conflict of Interest Statement}

The authors have no conflicts of interest to declare.

\section{Funding Sources}

No specific funding was received for this study.

\section{Author Contributions}

M.K., S.K., A.M. invented and designed the project. A.M., P.H.N., and A.W. performed the surgeries and extraction of human tissues. A.M. and M.K. performed the tissue processing procedures and generated the frozen sections. A.M. performed the histological stainings. S.K. optimized and performed the immunofluorescence stainings. M.K. took and edited the microscope pictures and created the Figures and schemata. S.K. and M.K. wrote the manuscript. A.W., A.F.M., P.H.N. helped in discussion of the project and performed proofreading of the manuscript. M.K., A.K. and S.L. were proofreading of the manuscript. Financial support was provided by S.L.

\section{Acknowledgment}

This study has been published on pre-print server [Klingenstein et al., 2020]. 


\section{References}

Au WW, Treloar HB, Greer CA. Sublaminar organization of the mouse olfactory bulb nerve layer. J Comp Neurol. 2002;446(1):68-80.

Bilinska K, Jakubowska P, Von Bartheld CS, Butowt R. Expression of the SARS-CoV-2 Entry Proteins, ACE2 and TMPRSS2, in Cells of the Olfactory Epithelium: Identification of Cell Types and Trends with Age. ACS Chem Neurosci. 2020;11(11):1555-62.

Brann DH, Tsukahara T, Weinreb C, Lipovsek M Van den Berge K, Gong B, et al. Non-neuronal expression of SARS-CoV-2 entry genes in the olfactory system suggests mechanisms underlying COVID-19-associated anosmia. Sci Adv.2020;6(31):eabc5801.

Caggiano M, Kauer JS, Hunter DD. Globose basal cells are neuronal progenitors in the olfactory epithelium: a lineage analysis using a replication-incompetent retrovirus. Neuron. 1994 13(2):339-52.

Chen M, Shen W, Rowan NR, Kulaga H, Hillel A, Ramanathan M, et al. Elevated ACE2 expression in the olfactory neuroepithelium: implications for anosmia and upper respiratory SARS-CoV-2 entry and replication. Eur Respir J. 2020;56:2001948.

Choi R, Goldstein BJ. Olfactory epithelium: Cells, clinical disorders, and insights from an adult stem cell niche. Laryngoscope Investig Otolaryngol. 2018;3(1):35-42.

Deffner F, Scharr M, Klingenstein S, Klingenstein M, Milazzo A, Scherer S, et al. Histological Evidence for the Enteric Nervous System and the Choroid Plexus as Alternative Routes of Neuroinvasion by SARS-CoV2. Front Neuroanat. 2020;14(74):596439.

Escada PA, Lima C, da Silva JM. The human olfactory mucosa. Eur Arch Otorhinolaryngol. 2009;266(11):1675-80.

Fan C, Li K, Ding Y, Lu WL, Wang J. ACE2 Expression in Kidney and Testis May Cause Kidney and Testis Damage After 2019-nCoV Infection. medRxiv. 2020, 20022418.

Fletcher RB, Das D, Gadye L, Street KN, Baudhuin A, Wagner A, et al. Deconstructing Olfactory Stem Cell Trajectories at Single-Cell Resolution. Cell Stem Cell. 2017;20(6):817$830 \mathrm{e} 8$.

Getchell ML, Getchell TV. Fine structural aspects of secretion and extrinsic innervation in the olfactory mucosa. Microsc Res Tech. 1992; 23(2):111-27.

Guan WJ, Ni ZY, Hu Y, Liang WH, Ou CQ, He JX, China Medical Treatment Expert Group for, C. Clinical Characteristics of Coronavirus Disease 2019 in China. N Engl J Med. 2020; 382(18):1708-20.
Hoffmann M, Kleine-Weber H, Schroeder S, Krüger N, Herrler T, Erichsen S, et al. SARSCoV-2 cell entry depends on ACE2 and TMPRSS2 and is blocked by a clinically proven protease inhibitor. Cell. 2020;182(2):271-280e.8.

Hopkins C, Surda P, Whitehead E, Kumar BN. Early recovery following new onset anosmia during the COVID-19 pandemic - an observational cohort study. J Otolaryngol Head Neck Surg. 2020;49(1):26.

Hou YJ, Okuda K, Edwards CE, Martinez DR, Asakura T, Dinnon KH, et al. SARS-CoV-2 Reverse Genetics Reveals a Variable Infection Gradient in the Respiratory Tract. Cell. 2020; 182(2):429-446e.14.

Klingenstein M, Klingenstein S, Neckel PH, Mack AF, Wagner A, Kleger A, et al. Evidence of SARS-CoV2 entry protein ACE2 in the human nose and olfactory bulb. BioRxiv. 2020 204602.

Kosugi EM, Lavinsky J, Romano FR, Fornazieri MA, Luz-Matsumoto GR, Lessa MM, et al. Incomplete and late recovery of sudden olfactory dysfunction in COVID-19. Braz J Otorhinolaryngol. 2020;86(4):490-96.

Krajewska J, Krajewski W, Zub K, Zatoński T. COVID-19 in otolaryngologist practice: a review of current knowledge. Eur Arch Otorhinolaryngol. 2020;277(7):1885-97.

Lechien JR, Chiesa-Estomba CM, De Siati DR, Horoi M, Le Bon SD, Rodriguez A, et al. Olfactory and gustatory dysfunctions as a clinical presentation of mild-to-moderate forms of the coronavirus disease (COVID-19): a multicenter European study. Eur Arch Otorhinolaryngol. 2020;277(8):2251-61.

Lee DJ, Lockwood J, Das P, Wang R, Grinspun E, Lee JM. Self-reported anosmia and dysgeusia as key symptoms of coronavirus disease 2019 . CJEM. 2020;22(5):595-602.

Lee Y, Min P, Lee S, Kim S-W. Prevalence and Duration of Acute Loss of Smell or Taste in COVID-19 Patients. J Korean Med Sci. 2020; 35(18):e174.

Maddux JF, Vogtsberger KN, Desmond DP, Esquivel M. Program changes and retention on methadone. J Subst Abuse Treat. 1993;10(6): 585-8.

Miller ML, Andringa A, Evans JE, Hastings L. Microvillar cells of the olfactory epithelium: morphology and regeneration following exposure to toxic compounds. Brain Res. 1995; 669(1):1-9.
Nagayama S, Homma R, Imamura F. Neuronal organization of olfactory bulb circuits. Front Neural Circuits. 2014;8:98.

Nagnan-Le Meillour P, Joly A, Le Danvic C, Marie A, Zirah S, Cornard JP. Binding Specificity of Native Odorant-Binding Protein Isoforms Is Driven by Phosphorylation and O-N-Acetylglucosaminylation in the Pig Sus scrofa. Front Endocrinol (Lausanne). 2019;9:816.

Ren X, Wang S, Chen X, Wei X, Li G, Ren S, et al. Multiple expression assessments of ACE2 and TMPRSS2 SARS-CoV-2 entry molecules in the urinary tract and their associations with clinical manifestations of COVID-19. Infect Drug Resist. 2020;13:3977-90.

Renne RA, Gideon KM, Harbo SJ, Staska LM, Grumbein SL. Upper respiratory tract lesions in inhalation toxicology. Toxicol Pathol. 2007;35(1):163-9.

Scott JW, Wellis DP, Riggott MJ, Buonviso N. Functional organization of the main olfactory bulb. Microsc Res Tech. 1993;24(2):142-56.

Steinke A, Meier-Stiegen S, Drenckhahn D, Asan E. Molecular composition of tight and adherens junctions in the rat olfactory epithelium and fila. Histochem Cell Biol. 2008;130(2): 339-61.

Suzuki Y, Takeda M, Farbman AI. Supporting cells as phagocytes in the olfactory epithelium after bulbectomy. J Comp Neurol. 1996; 376(4):509-17.

Suzuki Y, Takeda M, Obara N, Suzuki N, Takeichi $\mathrm{N}$. Olfactory epithelium consisting of supporting cells and horizontal basal cells in the posterior nasal cavity of mice. Cell Tissue Res. 2000;299(3):313-25.

Ueha R, Kondo K, Kagoya R, Shichino S, Ueha S, Yamasoba T. Background mechanisms of olfactory dysfunction in COVID-19: expression of ACE2, TMPRSS2, and Furin in the nose and olfactory bulb in human and mice. bioRxiv. 2020:097352.

Vogt RG, Rogers ME, Franco MD, Sun M. A comparative study of odorant binding protein genes: differential expression of the PBP1GOBP2 gene cluster in Manduca sexta (Lepidoptera) and the organization of OBP genes in Drosophila melanogaster (Diptera). J Exp Biol. 2002;205(Pt 6):719-44.

Zhou P, Yang XL, Wang XG, Hu B, Zhang L, Zhang W, et al. A pneumonia outbreak associated with a new coronavirus of probable bat origin. Nature. 2020;579(7798):270-3. 